\title{
design
}

\section{La convergencia de disciplinas hacia una nueva forma de hacer ciudad}

vol 12 / Jun.2015 31-48 pp Recibido: 23-11-2014 - revisado 23-12-2014 - aceptado: 15-01-2015 


\section{URBAN INTERACTION DESIGN \\ CONVERGENCE OF DISCIPLINE TOWARDS A NEW WAY OF \\ CITY-MAKING}

\section{ABSTRACT}

In a time of crisis in traditional urban policies and the generalization of a complex digital infrastructure in the networked society, the role of citizens stands as a crucial debate. While certain discourses are presented as irreversible and unequivocal on this relationship between urban living and technology, the article discusses the possibility of building alternative discourses from different urban interaction design practices that are converging in urban spaces are spaces for city-making and civic engagement .

\section{Keywords}

Urban media, smart cities, digital technologies, urban planning, activism.

\section{RESUMEN}

En un momento de crisis del modelo tradicional de las políticas urbanas y coincidiendo con la generalización de una compleja infraestructura digital en la sociedad conectada, el papel de la ciudadanía se sitúa como gran debate. Si bien determinados discursos se presentan como irreversibles y unívocos respecto a esta relación entre vida urbana y tecnología, el artículo plantea la posibilidad de construir discursos alternativos a partir de diferentes prácticas de diseño de interacción urbana que están confluyendo en el espacio urbano como espacio para construir ciudad y ciudadanía.

\section{Palabras Clave}

Urban media, ciudades inteligentes, tecnologías digitales, urbanismo, activismo. 


\section{INTRODUCCIÓN}

La forma de hacer ciudad es un proceso en crisis. Diferentes factores, desde cambios sociales, crisis de la política o transformación tecnológica, están contribuyendo a la materialización de un conflicto entre las formas institucionales de gobernar y dar forma a las ciudades y las expectativas de la ciudadanía sobre el papel que quiere tener en la ciudad que vive. Son muchos los movimientos sociales, las disciplinas académicas, los proyectos o las teorías que están contribuyendo en los últimos tiempos a la formación de un nuevo enfoque sobre cómo hacer ciudad que vaya más allá de las instituciones públicas y del urbanismo como instancias centrales o únicas de hacer ciudad.

El presente artículo plantea este punto de partida como escenario del cual emerge una disciplina naciente, a caballo entre el activismo, el arte y la cultura y la gestión urbana. Esta disciplina es el urban interaction design.

El origen de este artículo es, además, muy particular. Se alimenta directamente de una experiencia-experimento realizado en marzo de 2014 en el marco del proyecto Urbanlx $D^{1}$. Durante cinco días, un grupo de ocho investigadores con diferentes bagajes (algunos más cercanos al mundo académico, otros más al mundo del activismo; algunos más cercanos a la experimentación digital y las ciencias de la computación, otros más cercanos a los estudios urbanos) nos reunimos en un lugar aislado en Alemania para intentar definir este nuevo escenario y los contornos del urban interaction design. Siguiendo la metodología Booksprint ${ }^{2}$, de aquella intensa experiencia de debate, análisis y escritura colectiva surgió un texto ${ }^{3}$, Urban interaction design. Towards city making ${ }^{4}$, que es la base del presente artículo. Dicho texto (Fig.1) representa un acercamiento a la convergencia de disciplinas que están actuando en torno a la ciudad y su relación con la sociedad digital con una preocupación común a todas ellas: la apropiación de la tecnología para ampliar las capacidades de actuación de individuos, colectivos y comunidades en la construcción de las ciudades.

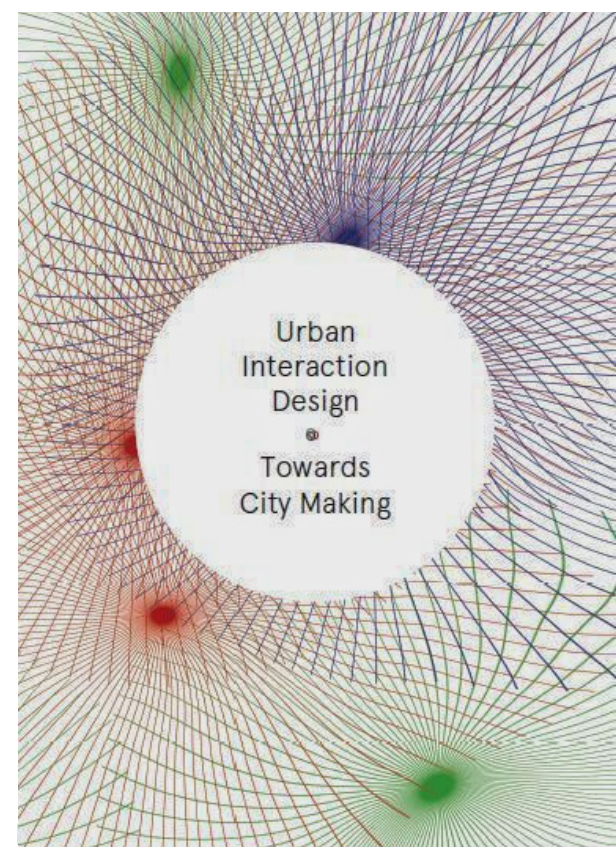

Figura 1. Portada del libro. Urban Interacion Design: towards city making.

(UrbanlxD 2014a) 
Aunque de perfiles muy difusos y sometida a diferentes interpretaciones e intentos de definición, la idea de smart city parece haber triunfado como término que pretende aglutinar la relación entre las tecnologías contemporáneas y su despliegue en la ciudad ${ }^{5}$. Sin entrar en su

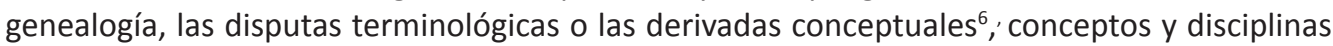
conexas -como urban computing, urban informatics, ubiquitous computing, ambient informatics, pervasive computing, locative media, physical computing, tangible media, situated technologies,...- comparten una serie de tecnologías y aplicaciones ${ }^{7}$. Todo ello ha ido dando forma a lo que, en una afortunada expresión, Adam Greenfield denominó Everyware (2006). Este escenario se define por la disolución de los ordenadores ${ }^{8}$ tal como los hemos conocido durante la primera etapa de la computación hasta el punto de confundirse con el propio entorno como espacio en el que la mediación y el procesado de la información acontece, ya sea en el espacio público, en los edificios, las infraestructuras, etc. Kitchin y Dodge (2011), con sus matices, han denominado a este escenario el híbrido Code/Space, una suerte de espacio nuevo conformado por dos categorías que hasta ahora funcionaban por separado (el espacio físico y el código informacional) y que ahora sólo pueden ser entendidas de manera unitaria para comprender el significado concreto para la experiencia humana de esta coexistencia. La generalización de tecnologías móviles y otras conexas han dado forma al mundo post-PC tal como lo imaginó Mark Weiser (Greenfield, 2006:11) y que implica la colonización de la vida diaria por las tecnologías de la información. Esta evolución tiene, además, una plasmación espacial como producción socio-material con una fuerte influencia en la vida cotidiana (Kitchin y Dodge 2011:13) y prácticamente cualquier hecho urbano está mediatizado por código de software.

Em este panorama, el posicionamiento discursivo (régimen discursivo ${ }^{9}$ ) de la smart city ha incorporado elementos conceptuales con suficiente fuerza como para situar las políticas públicas en un punto crítico, a caballo entre la epopeya por someter las supuestamente caóticas e ineficientes ciudades a unas reglas de control automatizado que harán posible su funcionamiento eficiente y racional. Esta formulación, presente de manera explícita y muchas veces simplemente implícita en gran parte del discurso mediático y político alrededor de la ciudad inteligente encierra importantes cuestionamientos sobre la forma en la que se propone gobernar la esfera pública y la manera en la que se enmarcará también la esfera de relación privada y social en la ciudad. Para algunos autores (Vanolo 2014), estamos ante un proceso de infiltración de unos determinados conceptos y prácticas institucionales que utilizan el reclamo de la smart city como "código de conducta" de la nueva "governmentality" ${ }^{10 " ~ h a c i a ~ l a ~ q u e ~ s e ~}$ dirige la política urbana.

Se trata, de hecho, de una cuestión que al menos en la última década ha ido ganando posiciones en la agenda de investigación urbana, moviéndose inicialmente desde el optimismo tecnológico sobre el potencial de la computación ubicua hasta la distopía crítica sobre sus consecuencias más negativas:

Industries and governments began heralding the coming "Internet of Things," where the global supply chain would be managed in ways that could create "smart objects" or a web-presence for consumer goods. Again popular media tended to focus on the surveillance possibilities, and ubiquitous or pervasive computing discourse began to take on a distinctly dystopian tone. (Galloway 2008:10) 
Es precisamente el trabajo de Adam Greenfield (2006) uno de los primeros en abordar de forma sistemática el impacto de la generalización de las tecnologías ubicuas en la vida urbana. A través de una serie de tesis, Greenfield disecciona el significado de estas tecnologías desde una perspectiva del comportamiento del usuario de las mismas (la ciudadanía, al fin y al cabo), y plantea una serie de afirmaciones que el imaginario de la smart city ha obviado prácticamente. La inevitabilidad de estar expuesto a muchas de estas tecnologías, en condiciones de desconocimiento, falta de voluntariedad o inconsciencia, teniendo en cuenta sus implicaciones en términos de privacidad, seguridad o igualdad, plantea serios interrogantes que caen necesariamente en la esfera de la política pública y cómo se define por los diferentes agentes que actúan sobre ella. Porque, a pesar de su inevitabilidad, existen diferentes alternativas en su diseño y no existe un relato único o una manera exclusiva de desplegarse (Greenfield, 2006:121).

Con ello, apuntamos aquí un primer elemento crítico para pensar las nuevas formas de hacer ciudad: en un escenario de creciente instrumentación tecnológica del funcionamiento de la ciudad, e igualmente en un momento de renovación del discurso sobre las políticas urbanas dominado por el escenario de la smart city, emerge una disponibilidad de opciones discursivas y prácticas más allá de un supuesto determinismo tecnológico.

\section{LA CIUDAD INSTRUMENTADA. NI IRREVERSIBLE NI UNÍVOCA}

Dicho todo lo anterior, el elemento subyacente en la narrativa de la ciudad inteligente es la ficción de la suficiencia tecnológica, una suerte de tecno-optimismo que protagoniza el ambiente alrededor de las smart cities. Todas las soluciones y propuestas sitúan los servicios o productos tecnológicos como la respuesta adecuada a los problemas que enfrentan las administraciones locales: dificultad en la escalabilidad de sus servicios, crecientes costes para los servicios públicos, pérdidas de eficiencia en las redes de infraestructuras, falta de interoperabilidad en los servicios, presión para personalizar los servicios públicos y adecuarlos a la demanda en tiempo real, etc.

Ante esta situación, la tentación de reducir todo a una respuesta tecnológica es evidente, pero exige preguntarnos cuánta tecnología es suficiente, en qué parte del ciclo de gestión de un servicio público es la tecnología el punto crítico o cuál es el nivel adecuado de tecnología que la ciudadanía puede y quiere utilizar en su vida diaria, tanto en su relación con la administración como para sus propias relaciones privadas y sociales.

De esta forma, siguiendo a Greenfield (2013), el régimen discursivo de la smart city presenta la tecnología como un objeto genérico. La forma de nombrar los nuevos productos o servicios que equiparán la smart city se alimenta de conceptos vagos, suficientemente atractivos pero inespecíficos, de manera que los catálogos comerciales conciben soluciones de "realidad aumentada", "muros interactivos", "monitorización inteligente", "soluciones de salud a distancia", etc. Sin embargo, lejos de ser una concesión al lenguaje comercial, necesariamente directo y reacio a las especificaciones técnicas, este recurso reproduce el fetichismo tecnológico de presentar tipologías genéricas de soluciones disponibles en el mercado, negando al debate la capacidad de discernir sobre sus usos concretos en determinados contextos, sobre las diferentes opciones de funcionamiento, las diferentes alternativas para financiarlos o las 
múltiples formas en las que su despliegue puede afectar a la ciudadanía. Con ello, se consigue centrar la tecnología como recurso "totémico" suficiente para imaginar la ciudad inteligente, dejando como una cuestión menor el viejo adagio "el diablo está en los detalles".

Frente a este optimismo tecnológico, incluso desde las propias ciencias de la computación ubicua es fácil encontrar visiones más equilibradas sobre el potencial real de lo tecnológico en relación con el resto de cuestiones que tienen que ver con la vida en la ciudad. Así lo afirma Gene Becker ${ }^{11}$ (mencionado por Townsend 2013:113):

Ubicomp is hard, understanding people, context, and the world is hard, getting computers to handle everyday situations is hard, and expectations are set away too high. I used to say ubicomp was a ten-year problem; now I'm starting to think that it's really a hundredyear problem

Los ejemplos podrían seguir: el potencial del open data frente a la transformación no puramente tecnológica que implica darle soporte bajo estrategias de open government, el potencial de la automatización del parking en superficie frente al modelo de movilidad que promueva una ciudad, etc. De hecho, podríamos atrevernos a decir que la tecnología es casi irrelevante en el éxito de las smart cities, de la misma manera que en muchas decisiones cotidianas de nuestra vida en la ciudad, las tecnologías asociadas al transporte o a la seguridad son mucho menos decisivas a la hora de acabar cogiendo a tiempo el metro o sentirnos seguros en una plaza que factores como el cuidado de lo común, la atención a las necesidades de las personas que están a nuestro alrededor, etc. Así, la inevitabilidad de las smart cities remite a la irrreversibiliddad tecnológica. Cualquier progreso humano parece quedar supeditado a la introducción de nuevos factores tecnológicos sin los cuáles, soluciones imaginativas a los problemas de las ciudades no serían posibles. Hill (2012:130) propone un caso ilustrativo para entender este supuesto. Frente al modelo del coche sin conductor, basado en la automatización de la movilidad como solución de, entre otras cosas, la siniestralidad y los riesgos de la carretera, podemos contraponer el modelo de los espacios compartidos (shared space) propugnado por Hans Monderman, una suerte de regulación libre de las intersecciones viarias, en el que se confía en el criterio de los conductores, influenciados bajo un cuidado diseño urbano que promueve la conducción cuidadosa y atenta al resto de vehículos y personas, la siniestralidad se reduce drásticamente. De esta manera, nos encontramos también con un interrogante clave a la hora de afrontar qué tipo de relación individual y colectiva podemos favorecer a través de la tecnología.

Esta suficiencia tecnológica encierra, además, una consecuencia subyacente: la tecnología será suficiente porque la tecnología será infalible. En la construcción del discurso ha sido básico apoyarse en las soluciones que estarán disponibles, sin mencionar no sólo las problemáticas conceptuales -tal como estamos abordando aquí- sino tampoco los problemas técnicos que pueden encerrar. Se trata de una cuestión absolutamente crítica en términos de privacidad de los datos y de la identidad de los usuarios de los sistemas inteligentes, de seguridad física, de estabilidad de las infraestructuras, etc. Estas situaciones, en el mejor de los casos son señaladas como excepciones menores frente al normal funcionamiento de los sistemas (Townsend 2013). Como señala Greenfield (2013), esta irreversibilidad está cargada de conceptos profundos para los estudios de la tecno-ciencia sobre la objetividad, el conocimiento perfecto, etc. Estos elementos se encuentran detrás de los lemas y textos sobre la smart city que apelan a la capacidad de ofrecer soluciones definitivas, soluciones únicas soluciones perfectas, soluciones 
indiscutibles políticamente o soluciones perfectamente integradas. Se trata de recursos comunes en cualquier material que analicemos que ofrezca la visión más optimista sobre la smart city.

Este posicionamiento oculta cuestiones que pueden resultar obvias en nuestra vida diaria y para las ciencias sociales, pero que son sistemáticamente olvidadas. Cuestiones como la imprevisibilidad de los usuarios de los sistemas inteligentes ( $\dot{y} y$ si, en realidad, no actuamos de manera racional frente a la información que nos ofrecen los contadores inteligentes?), el pequeño margen de actuación individual que siempre le quedará a cualquier policía sometido al más estricto de los sistemas inteligentes de gestión del tráfico a la hora de no castigar determinadas infracciones, la falta de información relacionada entre sistemas (con el clásico ejemplo de la corporación RAND y sus propuestas, basadas en modelos de dinámica urbana en la década de los 70 del siglo pasado en Nueva York), los sesgos de información o conocimiento (refiriéndonos aquí al también principio clásico de Heinsenberg), la inevitable necesidad de aplicar razonamientos ideológicos más allá de la calidad estadística que informe dicha decisión, los errores de medición, etc.

\section{NUEVAS PRÁCTICAS URBANAS EN LA SOCIEDAD CONECTADA}

2.1 La ciudad como plataforma

La mejor manera de comprometer a la ciudadanía en el desarrollo de las smart cities es reconocer lo que ya está sucediendo. Muchos ejemplos se podrían mencionar de proyectos e iniciativas que se encajan en una esfera amplia de apropiación ciudadana de los medios digitales (De Lange y De Waal, 2012) y sirven para ilustrar lo que los renders no pueden: un creciente número de personas están trabajando en lugares reales con problemas reales para desarrollar herramientas reales con tecnologías que ya están disponibles. Existe aún excesivo interés en promesas altamente dependientes de infraestructuras y soluciones orientadas a resolver únicamente los problemas de las administraciones locales. Sin embargo, las reglas han cambiado de alguna forma en la sociedad digital: las personas pueden desarrollar proyectos transformadores con un catálogo de tecnologías y aplicaciones abiertas y colaborativas:

An opposing force of entrepreneurs, hackers, and "citizen hacktivists" are pursuing a different vision of the future city. Their pitch: urban data can support cities that are more democratic, more responsive, and more resilient. These do-it-yourself (DIY) urbanists take a lightweight approach to exploiting urban data. They use off-the-shelf components, open-source hardware and software, and cooperative strategies.

In the future, successful cities will need to integrate both models. Solutions will combine the scale of big platforms with citizen-driven innovations. To a degree, this integration is well under way, but urban leaders need to educate themselves and frame an agenda of openness, transparency, and inclusiveness. (Townsend, 2010)

Frente a la tentación de creer que las posibilidades de automatización del control y seguimiento de cualquier parámetro de la ciudad nos llevan a un escenario de objetivización de las decisiones sobre los diferentes aspectos de la vida urbana (decisiones sobre políticas de seguridad, de gestión del tráfico, de vivienda, de espacio público, etc.), la realidad es que nada de esto debería sustraer la necesidad del debate público sobre cuestiones cruciales. Urbanizar la tecnología 
significa, principalmente, asignar al desarrollo tecnológico una función instrumental para atender a las necesidades de un determinado contexto para alinearse con las condiciones locales. Por ello, el liderazgo de los proyectos de implantación no debería recaer nunca en el esfuerzo de ventas de las utilities, empresas y otros agentes con intereses comerciales en este mercado (Goodspeed 2014). Sin entrar ni siquiera en las dimensiones más globales sobre el control de internet y todas las dinámicas derivadas (desde el control de la privacidad por parte de los grandes operadores y de los propios gobiernos hasta las resistencias de los diferentes sectores industriales impactados por el cambio en los modelos de negocio), las preguntas y los debates siguen siendo los mismos: ¿para quién son las smart cities?, ¿quién las protagoniza?, ¿quién se queda fuera?, ¿promueven o no la inclusión o son sólo formas sofisticadas de perpetuación de las relaciones de poder establecidas?, ¿cómo salvaguardar lo público?, ¿y cómo salvaguardar lo común?, ¿cómo pueden favorecer modelos estables de implicación y participación ciudadana?

\subsection{La tecnología como espacio abierto de intervención urbana}

Revisado el discurso predominante en el escenario de la smart city, corresponde ahora a este capítulo plantear un imaginario tecnológico alternativo, no tanto desde un planteamiento dicotómico, sino para abordar otros escenarios posibles concurrente. Dicho escenario, de hecho, no es necesario dibujarlo aquí. Ya existe pero, según el marco conceptual que hemos planteado, dispone de recursos mucho más débiles. Y, sin embargo, existe y tiene la capacidad de encontrar su propio espacio.

La crítica a la smart city ha constituido un sólido argumentario ${ }^{12}$ y no queremos aquí reproducirlos, sino tratar de darles forma en un discurso organizado y sistemático. Para ello, el punto de partida es reconocer que las mismas tecnologías sobre las que se construye el discurso anteriormente diseccionado son también las protagonistas de usos, prácticas e infraestructuras diferentes más cercanas a contextos activistas, artísticos, culturales, etc. (Townsend 2013:9).

La generalización de los dispositivos móviles y la disponibilidad ya de una amplia experiencia en el uso de herramientas digitales para la interacción social han creado un nuevo marco de actuación cívica que multiplica la capacidad de la ciudadanía para intervenir en los asuntos comunes. De hecho, es esta tecnología la que menos tiempo ocupa en la narrativa de la smart city, dominada por su preferencia por tecnologías por llegar, tal como señala Greenfield $(2013)^{13}$. En los últimos años hemos asistido a la irrupción de un nuevo discurso en torno a la ciudad que se focaliza en la idea de las smart cities como vehículo de transformación de lo urbano. Se trata de un debate hasta cierto punto polarizado por visiones muy distantes unas de otras respecto al papel de la tecnología en la ciudad y que encierra, en último término, una visión particular de la ciudad, sus agentes, el espacio físico y el espacio de los flujos de las relaciones que se dan en el entorno urbano. En este sentido, prácticamente el único punto de unión en esta "batalla" por las smart cities reside en la asunción de que las tecnologías digitales implican un cambio de escenario sobre la forma en la que se organiza la vida en la ciudad, incidiendo en algunos casos sobre la mejora de la eficiencia en la gestión de los servicios públicos y las infraestructuras que soportan el funcionamiento urbano, mientras que en otros casos se incide en las tecnologías digitales como habilitadoras de una nueva acción colectiva con mayor autonomía de organización, creación y aportación sin pasar por los filtros de lo público para crear herramientas de intervención y organización comunitaria para actuar sobre los asuntos públicos. 
Como hemos planteado anteriormente, uno de los recursos básicos del régimen discursivo de la smart city es el de la irreversibilidad. Sin embargo, cualquier régimen discursivo, siguiendo a Foucault, puede fracturarse a través de los micro-circuitos del poder, de los resultados de la auto-regulación o de resistencias localizadas (Kitchin y Dodge 2011:19). En definitiva, a pesar de encontrarnos ante un régimen discursivo aparentemente sólido, jerarquizado y top-down, existen vías de planteamiento de escenarios y regímenes o imaginarios alternativos porque incluso en el caso del régimen más extensivo, no podrá nunca llegar a todos los lugares y en todos los momentos en la ciudad.

De esta forma, el concepto de apropiación de las herramientas sigue siendo clave en una nueva teoría de la acción colectiva basada en las tecnologías digitales en cualquiera de sus formas (De Lange y de Waal 2012) y en la construcción de procesos y dinámicas de interacción social desde el compromiso cívico y la acción comunitaria en las que lo digital juega el papel de canalizador de un proceso más complejo de activación de la participación social en los asuntos comunes de la ciudad. La web y todas las soluciones derivadas abren nuevas opciones para desarrollar herramientas de activismo y compromiso social que, sólo apenas unos años apenas podíamos imaginar. Ha abierto la posibilidad de diseñar instrumentos de relación social que nos hace más abiertos y colaborativos en entornos personalizables de participación. Este es, precisamente, el campo de actuación de diferentes prácticas que aquí denominamos urban interaction design:

(...) digital tools and new media are allowing communities to shape and support the formation of publics around specific issues, linking concern, voice, and access in a way that allows them to use the city as a vibrant platform from a bottom-up perspective. This, ultimately, is a large part of what urban interaction design aims to facilitate. (UrbanIxD 2014a:23)

La generalización de los dispositivos móviles ha hecho que la interacción con un espacio digital sea constante y diaria. Nuestra vida es un permanente camino de rastros digitales de la interacción con las dependencias públicas, con las máquinas canceladoras o cualquier otro punto de la esfera de objetos públicos conectados (deWaal 2013), con los servicios y aplicaciones de nuestros dispositivos móviles, con las tarjetas bancarias, etc. Los ciudadanos somos un continuo de datos, meros generadores pasivos de información que va quedando por el camino del uso de las redes sociales. Mariposas ${ }^{14}$ revoloteando de un tema a otro, de una aplicación a otra, sin una dirección clara y perdidos en la confusión.

Una respuesta a esta desazón errante del ser humano del siglo XXI es, precisamente, la posibilidad de crear soluciones en las que podamos ser activos generadores de información y recopiladores de datos para agregarlos conjuntamente a través de procesos de crowdsourcing, por ejemplo. Diferentes proyectos relacionados con problemáticas urbanas se han basado en este modelo, buscando la aportación colectiva de información, en especial a través de iniciativas de mapeado o simplemente utilizando aplicaciones móviles a través de las cuales los usuarios generan y comparten información. Sin embargo, el peligro reside en generar con ello esquemas de actuación en los que el ciudadano -el usuario de las aplicaciones o participante en los procesos basados en tecnologías móviles- tenga un papel excesivamente pasivo como mero recolector ${ }^{15}$ de información. Siguiendo este planteamiento, Iveson (2011) plantea algunas claves para vincular los dispositivos móviles y, en general, los diferentes instrumentos digitales, en procesos de compromiso ciudadano real más allá del peligro de asignar al ciudadano un papel de mero 
recolector de información siempre y cuando en su diseño se cuente con una estrategia activa de ofrecer un rol central a la ciudadanía. De esta manera, la acción colectiva en torno al activismo digital adquiere un potencial de politización para dar respuesta a problemas urbanos desde la pertenencia a la ciudad y la apropiación de las tecnologías, frente a modelos de utilización de las tecnologías digitales para meras estrategias de control -el caso más claro es el de las aplicaciones dirigidas a la seguridad o la identificación de actividades- o de responsabilización:

The sum of interactive technologies that are reshaping our societies is also transforming our aspirations and the possibilities for a broader and more active role in the way citizens and institutions interact with publics, governance, community problems, and the products we use, etc. Urban interaction design appears as a new framework to deal with this growing claim for involvement in the decisions that affect us at any level. As such, it contributes to a well established domain (the strong tradition of and experiences from participatory planning and design in any of its forms) of how to design mechanisms and processes that promote and give citizens access to being protagonists of the public policies and decision-making procedures that affect our everyday lives. In this sense, urban interaction design stands in an appropriate place to enrich the traditional tools, practices and mechanisms that have been designed in the last decades to deal with this concern. (UrbanlxD 2014a:25)

Bajo estas consideraciones, los medios digitales o urban media, como ejemplo paradigmático de nuevos recursos ciudadanos para la acción colectiva, se convierten en una vía más para romper el modelo tradicional de intermediación institucional del ciudadano, que ahora amplía su capacidad para intervenir y hackear el gobierno para crear soluciones colectivas fuera de los circuitos tradicionales. Surge así, la posibilidad de dar una orientación ciudadana al discurso predominante del papel de la tecnología en la ciudad desde un sentido de apropiación ciudadana de las posibilidades tecnológicas digitales en el diseño de servicios y herramientas digitales, un cambio de perspectiva basado en tres pilares: la ciudad como plataforma para unos datos que deberían ser de "propiedad" colectiva, los medios digitales como instrumentos de acción colectiva, cocreación y auto-organización y la capacidad de sumar a más actores y públicos a los temas de interés público.

La visión tecno-optimista tiene un engarce directo con una suerte de determinismo sobre la aplicación de las tecnologías smart en la ciudad, que idealmente funcionarían como una relación de causa-efecto permanente. Sin embargo, esta visión oculta la capacidad de modificación de esa relación que tienen quienes están sometidos al régimen del código en cualquier elemento de la ciudad (De Waal 2013). Desde esta perspectiva, diferentes autores, y en especial Bell y Dourish (2006) han apuntado la necesidad de transformar la agenda de investigación en la materia hacia el constructivismo social como el mejor esquema a través del cual comprender el uso real y práctico de las tecnologías en su contexto social:

Discutido el relato de la smart city y explicado su régimen discursivo y los efectos de su imaginario tecnológico, hemos clarificado la posibilidad de construir, pensar, desarrollar y hacer realidad otros imaginarios posibles, más allá de que hayamos presentado el storytelling de la smart city como hegemónico y pretendidamente consensual. En este sentido, y de forma breve, planteamos que es posible pensar y diseñar productos, servicios, infraestructuras, relaciones sociales, dispositivos, interfaces, arquitecturas administrativas, espacios urbanos,... de una 
manera diferente, de manera que se pueda influir en el desarrollo próximo de los espacios urbanos con desde una mirada diferente. O, como señalan Greenfield y Shepard (2007), "occupy the imaginary of technological development in order to infuence its impact on the urban environment".

Esta manera implica construir un imaginario complejo capaz de incorporar el conocimiento de las diferentes ciencias y áreas de conocimiento implicadas en entender el funcionamiento de las ciudades, la vida en comunidad y el comportamiento individual en todo lo que tiene que ver con vivir con otros. Una manera que implique pensar en las ciudades que vivimos hoy como espacios de esperanza y no como lugares de los que huir hacia nuevas utopías escapistas. Una manera que ofrezca a la ciudadanía capacidad para actuar hoy (Fernández 2013) y ahora con más libertad sin tener que esperar a un futuro próximo para encontrar el potencial de las tecnologías inteligentes. Una manera no simplista de proponer soluciones para la ciudad basadas en la discusión democrática sobre cuáles son esos problemas y cómo los vamos a abordar y no en el fetichismo tecnológico de soluciones para todo simplistas y descontextualizadas.

\section{URBAN INTERACTION DESIGN COMO ESPACIO DE ENCUENTRO DE PRÁCTICAS URBANAS}

Como señalábamos en la introducción, la inspiración del presente artículo reside en un trabajo desarrollado en 2014 que tuvo como resultado la publicación de Urban interaction design. Towards city making como plasmación de una serie de reflexiones sobre la convergencia de disciplinas en torno a las nuevas prácticas de hacer ciudad. Dicho trabajo asumía como punto de partida el hecho de que hacer ciudad ya no es únicamente un asunto de planificadores públicos o de urbanistas en sentido estricto:

\section{(...) what we have observed is that 'the making of the city' is no longer just their concern. And no longer do their methodologies, expertise, and theories suffice to address the complex issues of the 21st century networked city. That's why increasingly we see designers of all sorts, IT specialists, urban anthropologists, philosophers, $\mathrm{HCl}$ researchers, artists and sociologists teaming up in coalitions that up to a few years ago were unthinkable. (UrbanIxD 2014a:4)}

El diseño de interacción urbana se propone como referencia de diferentes métodos y aproximaciones a la realidad de la sociedad conectada que están confluyendo. Esta confluencia se refleja a nivel de métodos y técnicas de trabajo en la transdisciplinariedad (Fig. 2) como metodología de proyectos e intervenciones, perseguida ahora como forma de abordar las limitaciones de las técnicas propias de cada uno de los campos frente a la complejidad de la realidad urbana:

The feeling of inadequacy that stems from researcher/practitioner's inabilities to fully comprehend the complexity of the city, the existing conceptual disciplinary maps, and the limitations presented by the methods used within a singular disciplinary field, has impacted each discipline at different moments, prompting various reactions. (UrbanlxD 2014a:12) 


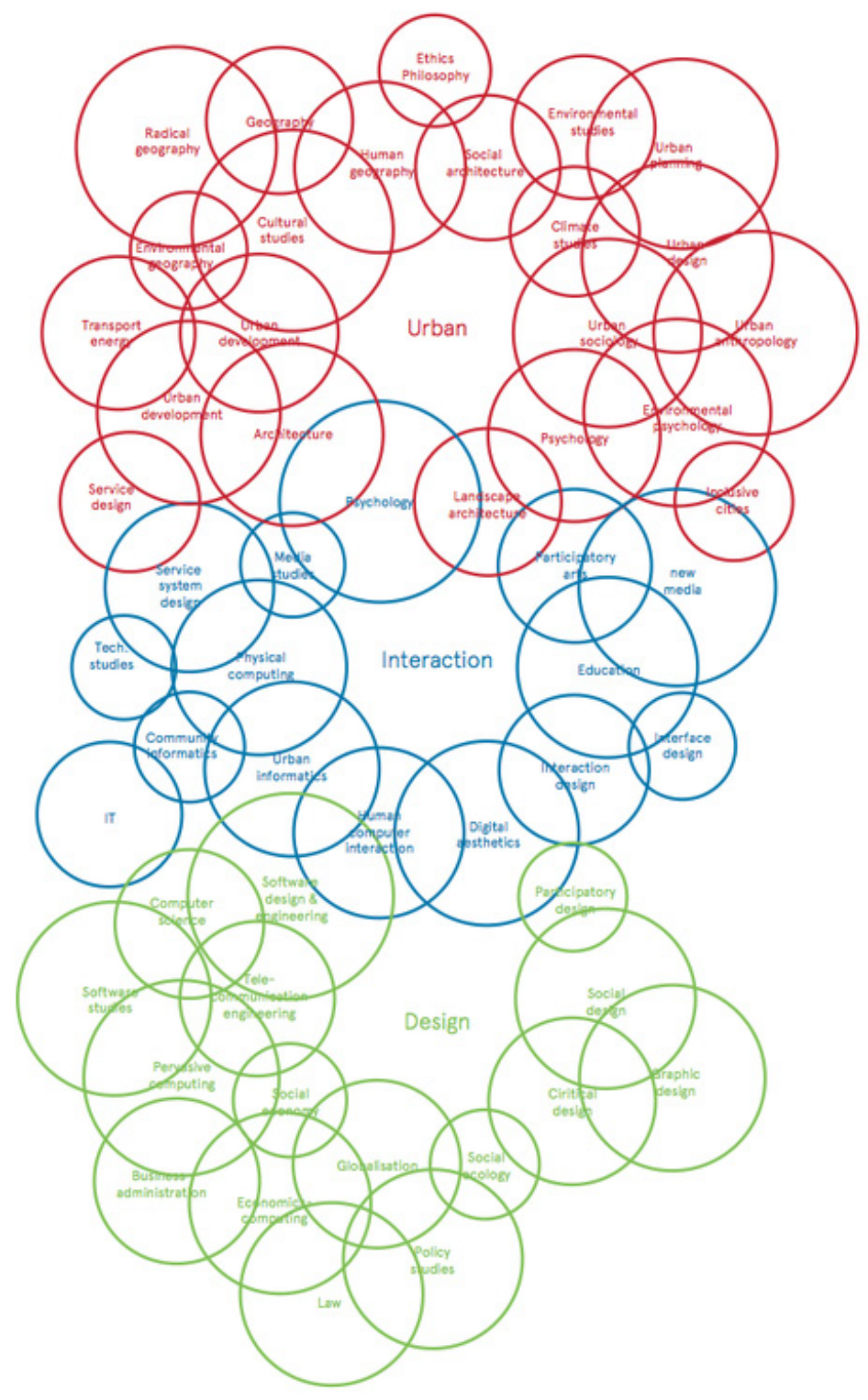

Figura 2. Urban Interacion Design: methods and approaches.

(UrbanlxD 2014a:14)

De esta forma, métodos como el mapeado ( $y$, en general, las técnicas cartográficas propias de los estudios urbanos), el prototipado (una aproximación propia del diseño) o el DIY (desde el mundo de la tecnología) son cada vez más utilizados de manera indistinta por unos y otros y forman parte de la caja de herramientas típica de diferentes proyectos de intervención urbana. Esta transdisciplinariedad trata de responder a las limitaciones de los diferentes campos de conocimiento y de acción para afrontar proyectos más integrales, complejos y multidimensionales. 
La complejidad de la sociedad conectada ha diluido de alguna forma los límites estancos de muchas disciplinas y, sobre todo, de muchas prácticas relacionadas con la ciudad. El diseño de interacción urbana es una respuesta a esa complejidad. Como señalaban Greenfield y Shepard (2007), campos como el diseño de interacción se han alimentado en los últimos tiempos de la contribución de otras disciplinas y campos de conocimiento, investigación y acción, conformando un nuevo espacio de encuentro multi-disciplinar.. Este espacio está formado por traiciones y metodologías que escapan del núcleo inicial de las comunidades del diseño de interacción, de las prácticas artísticas digitales o del activismo urbano. En cierta forma, es el ensanchamiento de cada una de estas disciplinas -y otras- el que marca los amplios horizontes de lo que aquí consideramos urban interaction design como lugar de encuentro del city-making de la ciudad contemporánea.

Some of the necessary insight can be supplied by people coming out of the user-experience (UX) community, but even there the focus has historically been on screen-based media. Some of it's going to come from people who's been through forma interaction-design programs. I daresay some of it's going to come from artists and psychologists and ethnographers -communities external to architecture or to urban planning proper. The professional tools and conventions and mindsets that will be necessary to meet this set of challenges, I can tell you from experience, barely even exist yet. (UrbanlxD 2014a)

En este sentido, estamos ante una renovación de la agenda de investigación y de acción en las tres aéreas básicas que estamos abordando (UrbanlxD 2014b):

- Lo urbano, como amplio contenedor de lo relacionado con la dimensión especial de las relaciones humanas

- Lo interactivo como referencia a unas tecnologías determinadas, aquellas relacionadas con la comunicación conectada.

- El diseño como referencia generica a las teorías y prácticas de construcción de experiencias y significados en objetos o procesos.

En cada una de estas tres grandes comunidades se está dando un proceso de salida de la zona de confort de las propias disciplinas y creándose nuevas relaciones, diálogos y prácticas (UrbanlxD 2014a:10):

(...) over the past few years we have seen a broad range of authors, institutions, organisations, projects, networks of practitioners and events who have started to explore territories beyond the comfort zone of their own disciplines because they see it as a necessary trajectory. To tackle complex issues in the networked city from a human and societal perspective, they have found it imperative to start to work together with partners across disciplinary and institutional boundaries.

Estas dinámicas están siendo impulsadas por diferentes tendencias en el diseño, organización e implementación de una nueva generación de proyectos de intervención en la ciudad con una aspiración clara: ampliar las capacidades de actuación de la ciudadanía como actores de la dinamización de la vida social en la ciudad, de la utilización de la ciudad como espacio de conflicto y negociación, de ampliación de los usos cívicos del espacio público de construcción creativa de la vida en la ciudad. En el terreno que nos ocupa, la dinámica más influyente ha 
sido la aparición de las lógicas amateur y la desprofesionalización de tecnologías y ámbitos de actuación supuestamente destinados a expertos profesionales en sentido estricto. La lógica DIY está en el ADN de muchas de las tecnologías que hoy forman parte del campo de acción del UIxD:

The disruption of clearly defined lines between who stands as active/passive, consumer/ producer and creator/producer is also the case for most of the disciplines involved in urban interaction designer. For example, what is an architect or an urban planner in a time when dynamic city building is gaining more and more traction? What is urban computing in a time when we have technologies available that make it increasingly easy to become an active maker of tangible devices that reshape the experience of urban life? And how can this not be political? What is design? Who is a designer? (UrbanlxD 2014a:47)

Todo ello enmarca un nuevo campo de acción e investigación en torno a la forma de hacer ciudad en la sociedad conectada y la ciudad instrumentada. Se trata de un enfoque que trabaja en la intersección y los espacios difusos de campos tradicionalmente separados, pero que cada vez disponen de más puntos de encuentro en la acción en la ciudad. Estas conexiones pasan por el trabajo transdisciplinar, el diseño crítico y el posicionamiento de los promotores de proyectos de diseño de interacción urbana como facilitadores dentro de las comunidades donde trabajan.

\section{Conclusiones}

En el artículo hemos planteado, de manera muy breve, una valoración narrativa sobre la propuesta discursiva de las smart cities, como principal concepto mediático utilizado para explicar el futuro de la ciudad contemporánea. Ante esta realidad, hemos tratado de confrontar algunos conceptos que apuntan a la irreversibilidad y a la ausencia de alternativas posibles para construir otro relato más significativo para la ciudadanía. Este relato, y sus prácticas asociadas, tiene más que ver con el presente, con los espacios y proyectos que ya están actuando hoy a través de tecnologías cotidianas, libres, sociales y accesibles.

De esta forma, planteamos una lectura cotidiana de la presencia digital en la ciudad, una lectura política de la forma en que esta presencia se despliega con consecuencias que están detrás de los diseños específicos de estas tecnologías, una lectura de la tecnología como construcción social y cultural y no como una serie de adelantos dados por un progreso no controlable por la ciudadanía. Para ello, ofrecemos la perspectiva del urban interaction design como una propuesta conceptual que pueda dar cabida a esta serie de prácticas, reflexiones y proyectos que juegan en la confluencia del arte, el activismo y la tecnología como forma emergente de hacer ciudad. 
Referencias

Bell, G. y Dourish, P. (2006). Yesterday's tomorrows: notes on ubiquitous computing's dominant vision. Personal Ubiquitous Computing (2006)

De Lange, M. y De Waal M. (2012). Ownership in the Hybrid City. Amsterdam: Virtueel Platform

De Waal, M. (2011a). The ideas and ideals in urban media theory. En Foth, M., Forlano, L., Satchell, C. y Gibbs, M. (eds). (2011). From Social Butterfly to Engaged Citizen: Urban Informatics, Social Media, Ubiquitous Computing, and Mobile Technology to Support Citizen Engagement, falta página Cambridge: MIT Press

De Waal, M. (2013). The city as interface. How new media are changing the city. Rotterdam: Nai 010

Fernández, M. (2013). Smart cities of the future. En Hemment, D. y Townsend A. (2013). Smart citizens. falta página Manchester: Future Everything

Foth, M., Forlano, L., Satchell, C y Gibbs, M. (eds.) (2011). From Social Butterfly to Engaged Citizen: Urban Informatics, Social Media, Ubiquitous Computing, and Mobile Technology to Support Citizen Engagement. Cambridge: MIT Press

Galloway, A. (2008). A Brief History of the Future of Urban Computing and Locative Media, (Tesis doctoral) Ottawa: Carleton University

Goodspeed, R. (2014). Smart cities: moving beyond urban cybernetics to tackle wicked problems. Cambridge Journal of Regions, Economy and Society Advance Falta volúmen revista número y página

Greenfield, A. (2006). Everyware: The Dawning Age of Ubiquitous Computing. Berkeley: New Riders Publishing

Greenfield, A. (2013). Against the smart city. Nueva York: Do Projects

Greenfield, A. y Shepard, M. (2007). Urban computing and its discontents, situated Technologies Pamphets 1. Nueva York: The Architectural League of New York 
Hill, D. (2012). The city that smart citizens built. City in a box Volume 34 falta página

Iveson, K. (2011). Mobile media and the strategies of urban citizenship: discipline, responsibilisation, politicisation, en Foth.M, Forlano, L., Satchell, C. y Gibbs, M. (eds). (2011). From Social Butterfly to Engaged Citizen: Urban Informatics, Social Media, Ubiquitous Computing, and Mobile Technology to Support Citizen Engagement. Cambridge: MIT Press

Kitchin, R. (2014). The Real-time City? Big Data and Smart Urbanism. Geo Journal 79. 1-14.

Kitchin, R. y Dodge, M. (2011). Code/Space. Software and Everyday Life. Cambridge: MIT Press,

Paulos, E. (2009). Manifesto of open disruption and participation. Recuperado de http://www.paulos.net/papers/2009/manifesto2009.html

Shepard, M. (2011). Sentient City. Ubiquitous Computing, Architecture, and the Future of Urban Space. Cambridge: MIT Press

Townsend, A. (2013). Smart Cities: Big Data, Civic Hackers, and the Quest for a New Utopia. New York: W. W. Norton \& Company.

URBANIXD (2014a). Urban interaction design. Towards city making. Amsterdam: UrbanIxD, faltan AUTORES

URBANIXD (2014b). Interactions in hybrid urban space: the UrbanIxD exhibition. Catalogue. Zelina (Croacia): UrbanlxD faltan AUTORES

Vanolo, A. (2014). Smartmentality: The Smart City as Disciplinary Strategy. Urban Studies 51(5) 2013, 1-16

Weiser, M. (1991). The computer for the 21st century, en Scientific American, September 1991 faltan páginas 
1. http://urbanixd.eu/

2. http://booksprints-for-ict-research.eu/

3. http://booksprints-for-ict-research.eu/2014/06/24/book-urban-interaction-designtowards-city-making/

4. Se puede acceder al texto aquí: http://issuu.com/urbanixd/docs/urbanixd_ towardscitymaking

5. Otros nombres con menos éxito mediático son señalados por Kitchin (2013): Cities which have embraced ICT as a development strategy, being pioneers in embedding digital infrastructure and systems into their urban fabric and utilising them for entrepreneurial and regulatory effect, have been variously labelled as 'wired cities' (Dutton et al., 1987), 'cyber cities' (Graham and Marvin 1999), 'digital cities' (Ishida and Isbister 2000), 'intelligent cities' (Komninos 2002), 'smart cities' (Hollands 2008) or 'sentient cities' (Shepard 2011). Whilst each of these terms is used in a particular way to conceptualise the relationship between ICT and contemporary urbanism, they share a focus on the effects of ICT on urban form, processes and modes of living, and in recent years have been largely subsumed within the label 'smart cities', a term which has gained traction in business and government, as well as academia.

6. Una de las aproximaciones conceptuales más claras y críticas se puede encontrar en De Waal, Martijn (2011) The ideas and ideals in urban media theory, en Foth, , Forlano, Satchell y Gibbs (eds.) (2011). From Social Butterfly to Engaged Citizen: Urban Informatics, Social Media, Ubiquitous Computing, and Mobile Technology to Support Citizen Engagement, MIT Press, Cambridge

7. Una lista aún más amplia de la mezcla de tecnologías, aplicaciones, servicios y conceptos teóricos es planteada por Galloway (2008:110): Ubiquitous Computing, Pervasive Computing, Mobile Computing, Smart Phones, Wearable Computing, Calm Technology, Spimes, Internet Protocol v6, Invisible Computing, Seamless Computing, Wi-Fi, Ambient Intelligence, Augmented Reality, Mixed Reality, Radio-Frequency Identification, Intelligent Environments, Internet-Of-Things, Physical Computing, Networked Objects, Smart Dust, Things That Think, Global Positioning System, Tangible Media, Mixed-Reality Games, Thinglinks, Body Area Networks, Blogjects, Context-Aware Computing, Cell ID, Spychips, Everyware, Participatory Panopticon, Smart Homes, Ambient Findability, Geospatial Web, Sensing Technologies, PhysicalMetaverse, Locative Media, Pervasive Play...

8. Otra formulación de interés es la relacionada con el concepto de "sentient city", desarrollado especialmente por Shepard (2011), que entiende la posibilidad de comprender la ciudad contemporánea digital como un espacio sensible (sentient). 
9. Utilizamos aquí el concepto de régimen discursivo tal como lo utilizan Kitchin y Dodge (2011: 19) a partir de Foucault, para quien un régimen discursivo-como el que defendemos que está detrás de las smart cities" es a set of interlocking discourses that sustain and reproduce, through processes of definition and exlusion, intelligibility and legitimacy, a particular set of sociospatial conditions. Such a regime provides the rationale for how sociosspatial relations are predominantly produced, legitimating the use of discursive and material practices that shape their production Para el caso del régimen discursivo o imaginario de la smart city, esto se traduce en una amalgama de razonamientos en torno a la seguridad, la eficiencia, la productividad, la fiabilidad, la flexibilidad, etc.

10. (...) the practices of subsumption of knowledge in the mechanisms of government, in the practices of 'the conduct of conduct', in the production of police systems (broadly understood as discipline systems) and in the cultural mechanisms that allocate specific identities to the ruler and the ruled. (Vanolo 2014:885)

11. Responsable de los primeros pasos de la empresa HP en materia de computación ubicua.

12. Véase gran parte de la bibliografía utilizada y, en especial, los trabajos de Townsend, Greenfield, de Waal, etc.

13. It is striking how poorly the dominant narrative seems to have accounted for broader trends in sociotechnical practice, chiefly the advent and widespread adoption of the smartphone. (...) it's positively weird how rarely these visions invoke the one piece of networked information technology that city dwellers all over the planet already have ready to hand. There's little need to invest in the comprehensive instrumentation of the urban fabric with sensors device controllers or informational displays when people themselves are already equipped with something that can act in all of these roles.

14. Véase FOTH, Marcus, Laura FORLANO, Christine SATCHELL y Martin GIBBS (eds.) (2011). From Social Butterfly to Engaged Citizen: Urban Informatics, Social Media, Ubiquitous Computing, and Mobile Technology to Support Citizen Engagement, MIT Press, Cambridge

15. Como decía Usman Haque en Surely there's a smarter approach to smart cities?:

We, citizens, create and recreate our cities with every step we take, every conversation we have, every nod to a neighbour, every space we inhabit, every structure we erect, every transaction we make. A smart city should help us increase these serendipitous connections. It should actively and consciously enable us to contribute to data-making (rather than being mere consumers of it), and encourage us to make far better use of data that's already around us.

En http://blog.cosm.com/2012/04/surely-theres-smarter-approach-to-smart.html 